OPEN ACCESS

Edited by:

Olivier Bernus,

Université de Bordeaux, France

Reviewed by:

Steve Poelzing,

Virginia Tech, United States Richard David Walton

Université de Bordeaux, France

${ }^{*}$ Correspondence: James Winter

J.Winter.1@bham.ac.uk

Specialty section:

This article was submitted to

Cardiac Electrophysiology, a section of the journal

Frontiers in Physiology

Received: 30 May 2019 Accepted: 26 September 2019

Published: 11 October 2019

Citation:

O'Shea C, Pavlovic D, Rajpoot K and Winter J (2019) Examination of the Effects of Conduction Slowing on the Upstroke of Optically

Recorded Action Potentials.

Front. Physiol. 10:1295.

doi: 10.3389/fphys.2019.01295

\section{Examination of the Effects of Conduction Slowing on the Upstroke of Optically Recorded Action Potentials}

\author{
Christopher O'Shea ${ }^{1,2}$, Davor Pavlovic ${ }^{1}$, Kashif Rajpoot ${ }^{3}$ and James Winter ${ }^{1 *}$ \\ ${ }^{1}$ Institute of Cardiovascular Sciences, University of Birmingham, Birmingham, United Kingdom, ${ }^{2}$ EPSRC Centre for Doctoral \\ Training in Physical Sciences for Health, University of Birmingham, Birmingham, United Kingdom, ${ }^{3}$ School of Computer \\ Science, University of Birmingham, Birmingham, United Kingdom
}

Introduction: The upstroke of optical action potentials (APs) recorded from intact hearts are generally recognized to be slower than those recorded with microelectrodes. This is thought to reflect spatial signal averaging within the volume of tissue that makes up the optical signal. However, to date, there has been no direct experimental study on the relationship between conduction velocity $(\mathrm{CV})$ and optical AP upstroke morphology in the intact heart. Notably, it is known that sodium channel block and gap junction inhibition, which both slow CV, exert differential effects on the upstroke velocity of microelectrode-recorded APs. Whether such differences are evident in optical APs is not known. The present study sought to determine the relationship between tissue CV and optical AP upstroke velocity in intact mouse hearts.

Materials and Methods: Isolated, perfused mouse hearts were stained with the potentiometric dye Rh-237. Fluorescent signals were recorded from across the anterior surface of the left and right ventricles during constant pacing. Maximum rate of change in fluorescence $\left(\mathrm{dF} / \mathrm{dt}_{\max }\right)$ and tissue $\mathrm{CV}$ were assessed in control conditions, during an acute period of low-flow ischemia, and following perfusion of flecainide ( $1-3 \mu \mathrm{mol} / \mathrm{L})$, a sodium channel blocker, or carbenoxolone (10-50 $\mu \mathrm{mol} / \mathrm{L})$, a gap junction inhibitor.

Results: During epicardial pacing, an anisotropic pattern was observed in both activation and $\mathrm{dF} / \mathrm{dt}_{\max }$ maps, with more rapid optical $\mathrm{AP}$ upstroke velocities orientated along the fastest conduction paths (and vice versa). Low-flow ischemia resulted in a time-dependent slowing of ventricular $\mathrm{CV}$, which was accompanied by a concomitant reduction in optical AP upstroke velocity. All values returned to baseline on tissue reperfusion. Both flecainide and carbenoxolone were associated with a concentrationdependent reduction in CV and decrease in optical AP upstroke velocity, despite distinct mechanisms of action. Similar responses to carbenoxolone were observed for low- (156 $\mu \mathrm{m}$ pixel with) and high- (20 $\mu \mathrm{m}$ pixel width) magnification recordings. 
Comparison of data from all interventions revealed a linear relationship between CV and upstroke $\mathrm{dF} / \mathrm{dt}$.

Conclusion: In intact mouse hearts, slowing of optical AP upstroke velocity is directly proportional to the change in CV associated with low-flow ischemia, sodium channel block, and gap junction inhibition.

Keywords: optical mapping, conduction velocity, action potential upstroke, anisotropic conduction, ventricular, electrophysiology - basic

\section{INTRODUCTION}

Cardiac optical mapping, using potentiometric dyes and fluorescent-light-sensitive digital cameras, allows researchers to study the electrophysiological properties of the heart at unparalleled spatial resolution. In intact heart tissue, the morphology of action potentials (APs) recorded with optical mapping typically exhibit reduced upstroke velocity and longer rise times compared to those recorded from the same tissues with microelectrode techniques (Morad and Salama, 1979; Girouard et al., 1996; Hyatt et al., 2003; Salama et al., 2005; Bishop et al., 2007) (though not all studies agree) (Windisch et al., 1985). Meanwhile, in isolated cardiac myocytes, rates of change for optical AP upstrokes have been reported to be similar to those recorded through microelectrodes (Warren et al., 2010). The slower optical AP upstroke in intact cardiac tissue is thought to reflect the photon scattering effects of the tissue (Bishop et al., 2007, 2009), as well as the rate of conduction of electrical waves within the myocardium (Morad and Salama, 1979; Girouard et al., 1996; Hyatt et al., 2003; Salama et al., 2005; Bishop et al., 2007). Optical signals are integrated from a volume of tissue in which there is asynchronous activation and where spatial averaging of signals across and through the tissue is thought to slur the AP upstroke (Morad and Salama, 1979; Girouard et al., 1996; Hyatt et al., 2003; Salama et al., 2005; Bishop et al., 2007). Indeed, computational modeling studies suggest that the rise time of optical AP in simulated human ventricle is a nonlinear function of tissue conduction velocity (CV); where slower $\mathrm{CV}$ equates to an increase in the time for tissue activation and therefore a slower AP upstroke (Hyatt et al., 2003). There has, however, been no experimental examination of the impact of conduction slowing on optical AP upstroke morphology in intact hearts.

Slowing of CV can occur through multiple mechanisms, including acute ischemia, tissue remodeling (hypertrophy and fibrosis), reduced sodium channel availability, and reduced gap junction coupling. Sodium channel block reduces the number of available sodium channels and decreases the transmembrane sodium current, which slows tissue CV. It is well established that concurrently with slowing $\mathrm{CV}$, sodium channel block reduces the rate of maximum AP depolarization $\left(\mathrm{dV} / \mathrm{dt}_{\max }\right)$ (Borchard and Boisten, 1982; Kojima et al., 1989; Ferrero et al., 2007). Meanwhile, gap junction inhibition slows tissue CV due to the reduction in current (source) flowing into neighboring myocytes

Abbreviations: $\mathrm{AP}$, action potential; $\mathrm{CV}$, conduction velocity; $\mathrm{dF} / \mathrm{dt}$, rate of change in fluorescence. (sink) (de Groot et al., 2003). Model studies suggest that gap junction uncoupling slows $\mathrm{CV}$, yet results in increased rate of AP depolarization contrasting with observations in the presence of sodium channel blockers (Spach et al., 1987, 1988). These predictions however, are inconsistently supported (Thomas et al., 2003; Poelzing and Rosenbaum, 2004; Dhillon et al., 2013) or disputed (Jalife et al., 1989; Ozaki et al., 1989; Rohr et al., 1998; de Groot et al., 2003; Entz et al., 2016) by experimental data using a variety of experimental models, techniques, and gap junction uncoupling interventions.

In principle, the rate of change of an optical AP upstroke is a function of (1) the intrinsic AP upstroke within each individual myocyte, (2) the activation delay across and through the tissue integrated by each camera pixel, and (3) the photon scattering effects of the tissue (which increases the volume of tissue that contributes to the signal) (Bishop et al., 2009). Whether any differential effects of ischemia, sodium channel block and gap junction inhibition would be evident in optically recorded APs is not known. The present study sought to determine the relationship between tissue $\mathrm{CV}$ and optical AP upstroke velocity at different fractional AP levels during acute ischemia, sodium channel block, and gap junction inhibition.

\section{MATERIALS AND METHODS}

\section{Animal Welfare}

All procedures were undertaken in accordance with ethical guidelines set out by the United Kingdom Animals (Scientific Procedures) Act 1986 and Directive 2010/63/EU of the European Parliament on the protection of animals used for scientific purposes. Studies conformed to the Guide for the Care and Use of Laboratory Animals published by the U.S. National Institutes of Health under assurance number A5634-01. Studies were approved by the University of Birmingham Welfare and Ethical Review Board.

\section{Optical Mapping \\ Mouse Hearts}

Male mouse (C57/BL6, 25-30 g, Charles River, United Kingdom) hearts were isolated under isoflurane induced anesthesia ( $4 \%$ in $100 \% \mathrm{O}_{2}$ ) with concomitant intraperitoneal injection of heparin (100 units injected 5-min before heart isolation). Hearts were retrogradely perfused via the aorta at a perfusion pressure of 70-80 $\mathrm{mmHg}$ with an oxygenated crystalloid buffer, containing (in $\mathrm{mM}$ ); $\mathrm{NaCl} 114, \mathrm{KCl} 4, \mathrm{CaCl} 1.4, \mathrm{NaHCO}_{3} 24, \mathrm{NaH}_{2} \mathrm{PO}_{4} 1.1$, 


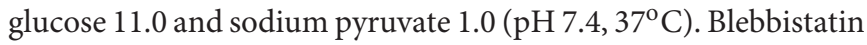
was added to the perfusate at a concentration of $15 \mu \mathrm{mol} / \mathrm{L}$ and solutions were continuously passed through a nitrocellulose filter ( $5 \mu \mathrm{m}$ pore diameter). Once contraction had abated, the potentiometric dye Rh-237 was loaded by injection into the perfusion line. $100 \mathrm{~mL}$ of stock solution (1.25 mg/mL in DMSO) was injected over a period of 5-min. Final DMSO concentration was $0.001 \%$. Hearts were illuminated at $530 \pm 25 \mathrm{~nm}$ and emitted light $>630 \mathrm{~nm}$ was collected via an Olympus MVX10 stereomicroscope and Evolve Delta $512 \times 512$ EMCCD camera. Images were taken from the anterior left and right ventricular surface. Unless stated, data was collected at an acquisition sampling rate of $1 \mathrm{kHz}$ with a pixel width of $156 \mu \mathrm{m}$.

During recordings hearts were paced with $1 \mathrm{~ms}$ pulses with a $110 \mathrm{~ms}$ cycle length from the epicardial surface at $4 \times$ the diastolic threshold using a bipolar pacing electrode (electrode spacing $\sim 1 \mathrm{~mm}$ ). Recording time was 5 -s. Hearts were subjected to interventions to alter ventricular CV. Namely, these were (i) 3-min of low-flow global ischemia (25\% of original flow rate) (ii) increasing concentrations of the sodium channel blocker flecainide (1-3 $\mu \mathrm{mol} / \mathrm{L}$, total perfusion time 30-45 $\mathrm{min}$ ) (iii) increasing concentrations of the gap junction inhibitor carbenoxolone (10-50 $\mu \mathrm{mol} / \mathrm{L}$, total perfusion time 30-45 $\mathrm{min}$ ). The pacing threshold was selected to ensure electrical capture during low-flow ischemia protocols.

\section{Data Processing}

Data processing was performed using an updated version of our freely available electrophysiological mapping software, ElectroMap ${ }^{1}$ (O'Shea et al., 2019), which is based in MatLab (The MathWorks). To improve the signal-to-noise of optical APs, 40-sequential beats were aligned and averaged. A region of interest encompassing the ventricles (left anterior and right) was selected and data were processed, unless otherwise stated, using a Gaussian spatial filter $[5 \times 5$ pixel area, standard deviation $(\sigma)=1]$. No temporal filtering was applied.

Action potential amplitude was normalized between 0 and 1 . Normalization is required because signal amplitude [and so differential fluorescent amplitude $(\mathrm{dF} / \mathrm{dt})]$ is affected by heterogeneities in dye loading and tissue illumination; which is a general limitation of single-wavelength fluorescent indicators. To quantify the AP upstroke morphology, we computed the maximum differential value of the optical AP upstroke $\left(\mathrm{dF} / \mathrm{dt}_{\max }\right)$. As described in previous studies, the fractional level that $\mathrm{dF} / \mathrm{dt}_{\max }$ occurs at was defined as $\mathrm{V}_{\mathrm{F}}{ }^{*}$ (Salama et al., 1994). In some studies, we also measured $\mathrm{dF} / \mathrm{dt}$ at different fractional levels between 0.1 and $0.9\left(\mathrm{dF} / \mathrm{dt}_{0.1}-\mathrm{dF} / \mathrm{dt}_{0.9}\right)$. Unless stated otherwise, cubic spline interpolation was applied to increase the effective sampling rate from 1 to $16 \mathrm{kHz}$. Local tissue activation times were measured as the time of the maximum upstroke velocity (i.e., time of $\mathrm{dF} / \mathrm{dt}_{\max }$ and $\mathrm{V}_{\mathrm{F}}{ }^{*}$ ) (Walton et al., 2012). CV was calculated from the isochronal maps using the polynomial multi vector method with a $5 \times 5$ pixel area (Bayly et al., 1998; O'Shea et al., 2019).

${ }^{1}$ https://github.com/CXO531/ElectroMap

\section{Analysis and Statistics}

Hearts with signs of thrombi or with an initial heart rate less than 250 beats per minute were terminated and excluded. Hearts were randomized to different treatments prior to the experiments, but the nature of the paired (within heart) experimental design meant that blinding was unfeasible. However, excluding the initial determination of the region of interest, data were processed automatically with the same analytical steps and without user input, thus limiting sources of bias and error. Data analysis and statistical analysis was performed in GraphPad Prism (v8, GraphPad Software, United States). In all data presented, the mean value of $\mathrm{dF} / \mathrm{dt}, \mathrm{CV}$ or $\mathrm{V}_{\mathrm{F}}{ }^{*}$ from the entire analyzed field of view was calculated for each individual heart. The mean data ( $n=1$ for each heart) was then used for statistical tests, i.e., $n=14$ hearts for ischemia-reperfusion, $n=7$ for flecainide/carbenoxolone treatment, respectively. Comparisons were paired (within experiment) and thus analysis was performed using paired $t$-tests, or repeated measures one- or two-way ANOVA with Bonferroni post hoc tests. Statistical significance was taken as $p<0.05$. Unless otherwise stated, data is presented as Tukey's boxplots.

\section{RESULTS}

\section{Relationship Between Optical AP Upstroke Velocity, Conduction Velocity, and $\mathbf{V}_{\mathbf{F}}{ }^{*}$}

$\mathrm{V}_{\mathrm{F}}{ }^{*}$ is the fractional amplitude at which the optical AP upstroke has its fastest rate of rise and is shown in previous studies to reflect the subsurface orientation of depolarizing wavefronts as they spread through the myocardium (Hyatt et al., 2003, 2005, 2008; Zemlin et al., 2008). Here we examine the relationship between $\mathrm{V}_{\mathrm{F}}{ }^{*}$, the maximum rate of change of the optical AP $\left(\mathrm{dF} / \mathrm{dt}_{\max }\right)$ and the pattern of electrical propagation in the intact heart. Figure 1 shows data from a representative mouse ventricle during epicardial pacing (point stimulation). Pacing was associated with an anisotropic pattern of conduction with fast (longitudinal) and slow (transverse) conduction paths. A typical oval isochronal map is shown in Figure 1A, where the faster conduction is indicated by the red arrow and the slowest conduction path by the black arrow. To the right is the corresponding $\mathrm{V}_{\mathrm{F}}{ }^{*}$ map in the same heart (Figure 1B). Consistent with previous reports, $\mathrm{V}_{\mathrm{F}}{ }^{*}$ was low (0.5 or less) along the longitudinal axis, and high (greater than 0.5) in the transverse direction (Hyatt et al., 2003, 2005, 2008; Zemlin et al., 2008).

Figure $1 \mathrm{C}$ shows the corresponding maximum rate of change of fluorescence $\left(\mathrm{dF} / \mathrm{dt}_{\max }\right)$ map. $\mathrm{dF} / \mathrm{dt}_{\max }$ was measured as the maximum of the first derivative of the normalized optical AP trace (signal amplitude normalized from 0 to 1). Faster rates of changes are observed to align along the faster, longitudinal, conduction path. Differences in the morphology of APs in longitudinal and transverse conduction paths are shown in the example traces in Figure 1D. In keeping with previous reports, the foot of the AP was shallow along the transverse conduction path, where $\mathrm{V}_{\mathrm{F}}{ }^{*}$ is high, and steep along the longitudinal 


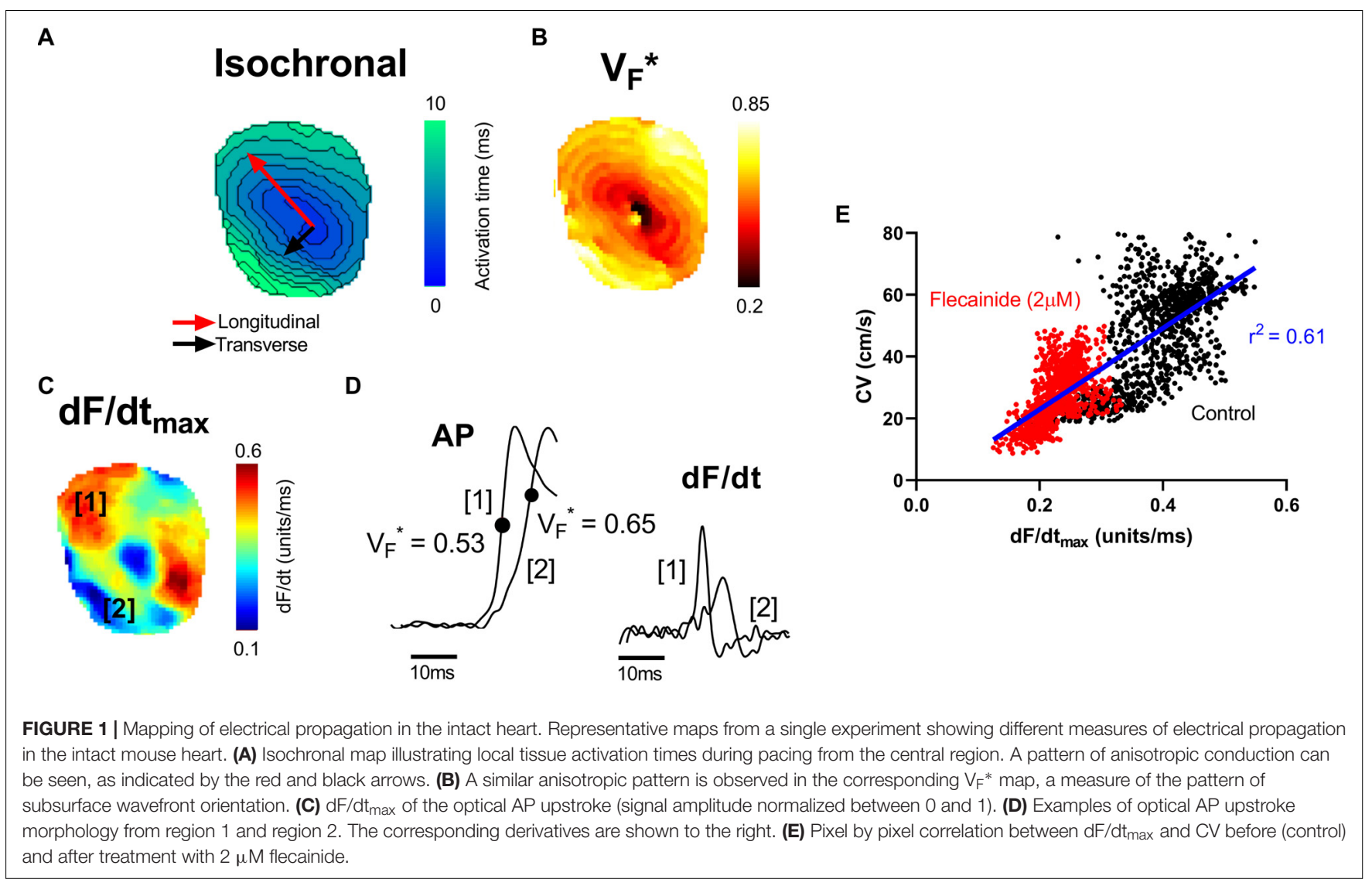

conduction path, where $\mathrm{V}_{\mathrm{F}}{ }^{*}$ is low (Hyatt et al., 2005). The corresponding $\mathrm{dF} / \mathrm{dt}$ traces derived from these example APs are shown to the right. Along the faster, longitudinal, path (region 1), a larger maximum $\mathrm{dF} / \mathrm{dt}$ value is present. Figure $1 \mathrm{E}$ shows the pixel to pixel correlation of local $\mathrm{CV}$ with $\mathrm{dF} / \mathrm{dt}_{\max }$ before and after treatment with $2 \mu \mathrm{M}$ flecainide in this representative mouse heart (see below for further results on the effect of flecainide). A positive linear correlation is observed, with faster local $\mathrm{CV}$ areas exhibiting larger $\mathrm{dF} / \mathrm{dt}_{\max }$.

\section{Impact of Conduction Slowing on Optical AP Upstroke Velocity}

The experiments described below were designed to quantify the effects of interventions that cause conduction slowing on the maximum rate of change of the optical AP upstroke $\left(\mathrm{dF} / \mathrm{dt}_{\max }\right)$ and at different fractional AP amplitudes $\left(\mathrm{dF} / \mathrm{dt}_{0.1}-\mathrm{dF} / \mathrm{dt}_{0.9}\right)$.

\section{Low-Flow Ischemia}

Data presented in Figure 2 summarize the effects of a short period of low-flow global ischemia (25\% of the initial flow-rate) and subsequent flow-restoration (reperfusion) on ventricular conduction in the perfused mouse heart. Reduced coronary perfusion results in the build-up of metabolic by-products, acidification of the cell, and the accumulation of potassium ions in the extracellular space; all of which drive increase in the resting membrane potential, reduce sodium channel availability and slow CV (Stanley, 2001; Klabunde, 2017). Representative activation maps during control conditions, during ischemia and following reperfusion are shown in Figure 2A. In the mouse heart, a short (3-min) period of low-flow ischemia was associated with a progressive slowing of ventricular $\mathrm{CV}$ as seen by a prolongation of activation time and the tightening of isochronal lines. Corresponding representative $\mathrm{dF} / \mathrm{dt}_{\max }$ maps are shown in Figure 2B. Slowing of CV was paralleled by a decrease in $\mathrm{dF} / \mathrm{dt}_{\max }$, indicative of a slowing of optical AP upstroke velocity. Data summarizing the temporal response in $\mathrm{dF} / \mathrm{dt}_{\max }$ from all areas of the heart are shown in Figure 2C (black). It is well established fractional value at which $\mathrm{dF} / \mathrm{dt}_{\max }$ occurs $\left(\mathrm{V}_{\mathrm{F}}{ }^{*}\right)$, is dependent on the transmural wave orientation, with $\mathrm{V}_{\mathrm{F}}{ }^{*} \sim 0.5$ reflecting parallel conduction to the epicardial surface (Hyatt et al., 2003, 2005, 2008; Zemlin et al., 2008). We hence repeated the analysis but restricted measuring $\mathrm{dF} / \mathrm{dt}_{\max }$ to pixels in which $0.45 \leq \mathrm{V}_{\mathrm{F}}{ }^{*} \leq 0.55$, Figure $2 \mathrm{C}$ (red). This did not significantly alter $\mathrm{dF} / \mathrm{dt}_{\max }$ response to ischemia-reperfusion.

The corresponding changes in mean tissue CV are shown in Figure 2D. Figure 2E shows the change in AP upstroke velocity during low flow ischemia at different fractional levels of the AP levels ranging from 0.1 to 0.9 . A decrease in $\mathrm{dF} / \mathrm{dt}$ due to ischemia is seen at all fractional levels of the AP, not just $\mathrm{dF} / \mathrm{dt}_{\max }$. On reperfusion, tissue $\mathrm{CV}$ and $\mathrm{dF} / \mathrm{dt}_{\max }$ gradually recovered to control values. In summary, ischemia was associated with a predictable and reversible slowing of $\mathrm{CV}$ that was paralleled by a decrease in the maximum rate of change of the optical AP upstroke, and at different fractional levels of the upstroke. 


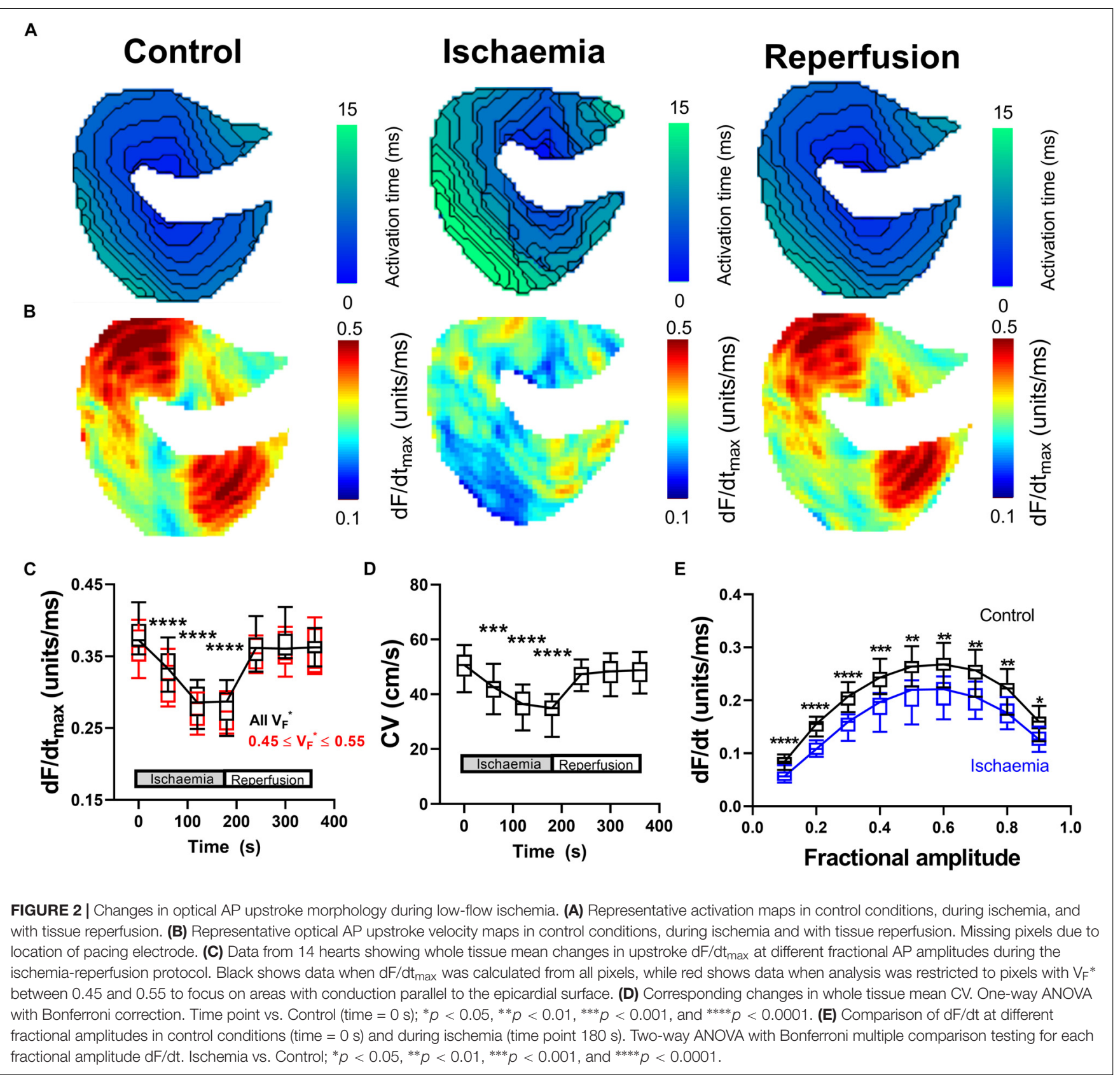

\section{Sodium Channel Block and Gap Junction Inhibition}

The effects of reducing sodium channel and gap junction availability/conductance were examined using flecainide, a sodium channel blocker, and carbenoxolone, a gap junction inhibitor. Data are presented in Figure 3. Both flecainide and carbenoxolone were associated with a concentration-dependent slowing of ventricular $\mathrm{CV}$ and corresponding reduction in $\mathrm{dF} / \mathrm{dt}_{\max }$. Typical AP recordings from a single pixel before and after drug perfusion are presented in Figures 3A,D for flecainide and carbenoxolone, respectively. Both drugs were associated with a decrease in amplitude-normalized $\mathrm{dF} / \mathrm{dt}$, as shown in the relative right-hand traces. Concentration-dependent slowing of $\mathrm{dF} / \mathrm{dt}_{\max }$ are shown in Figures $3 \mathbf{B}, \mathbf{E}$. The corresponding change in $\mathrm{CV}$ is shown in Figures 3C,F for flecainide and carbenoxolone, respectively.

\section{Dependence of Optical AP Upstroke Velocity on Tissue CV}

Figure 4A shows the relationship between tissue $\mathrm{CV}$ and maximum optical AP upstroke velocity, including data for lowflow ischemia, flecainide and carbenoxolone protocols. Figure 4A show that in spite of their differing mechanisms of action, all data points fall on a simple linear relationship, with no obvious separation in the responses to ischemia, flecainide and carbenoxolone. The same holds for $\mathrm{dF} / \mathrm{dt}$ measured at the foot $\left(\mathrm{dF} / \mathrm{dt}_{0.1}\right.$, Figure $4 \mathrm{~B}$ red) and head $\left(\mathrm{dF} / \mathrm{dt}_{0.9}\right.$, Figure $4 \mathrm{~B}$ black $)$ 


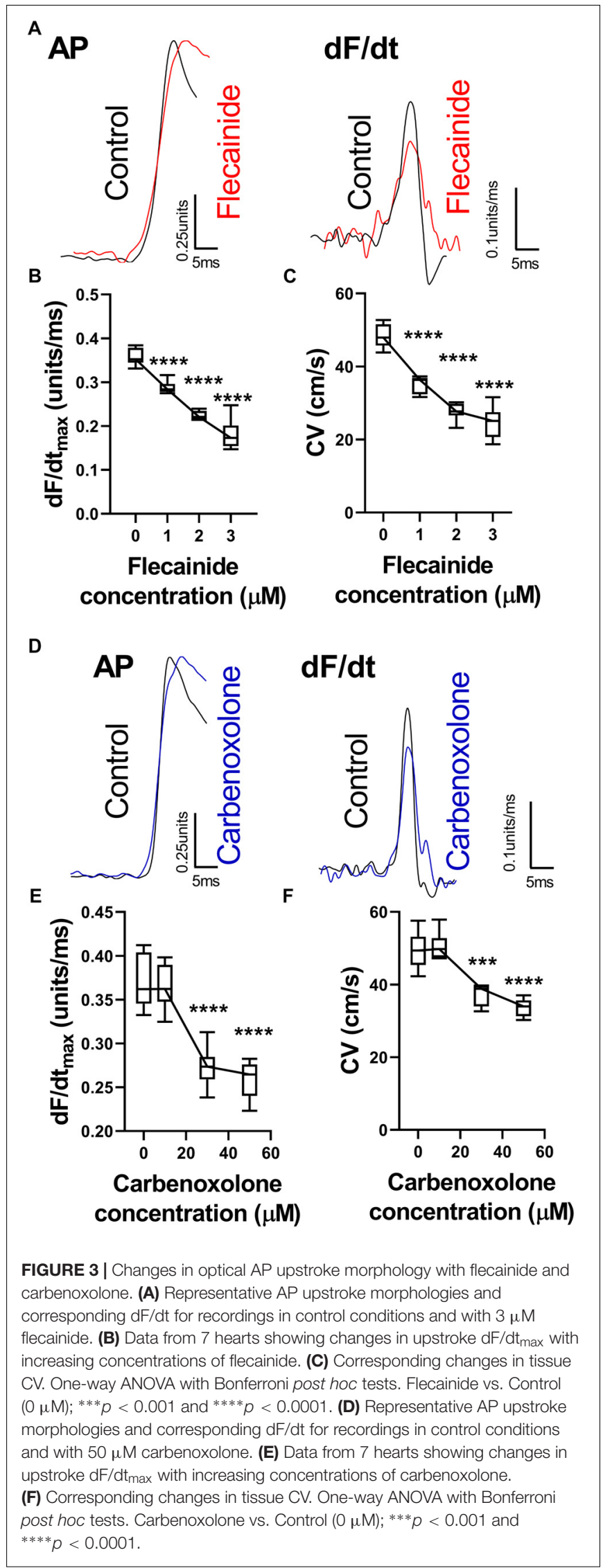

of the AP upstroke. A non-linear relationship between tissue CV and rise-time (defined as time between 10 and 90\% amplitude of the depolarization) of the optical AP was found, as shown in Figure 4C, where a non-linear exponential decay better matched the observed relationship.

\section{Influence of Sampling Rate and Spatial Resolution}

Figures 5A,B summarizes the effects of altering sampling frequency and spatial resolution (pixel width) on optical AP morphology in isolated perfused mouse hearts. Data presented in Figure 5A show a strong dependence on sampling rate, with a direct correlation between increases in acquisition sampling frequency and increased maximum optical AP upstroke velocity. Figure 5B shows the impact of post-acquisition pixel-binning on AP upstroke velocity in recordings made at low and highmagnification, equating to initial pixel widths of 156 and $20 \mu \mathrm{m}$, respectively. Binning pixels $(2 \times 2,3 \times 3 \ldots)$ led to a reduction in maximum optical AP upstroke velocity in recordings made with low-magnification (open circles), but not those recorded at higher-magnification (open squares) in the pixel width ranges tested. Figures 5C,D similarly summaries the results of altering acquisition sampling frequency and spatial resolution on the fractional level at which the optical AP exhibits $\mathrm{dF} / \mathrm{dt}_{\max }\left(\mathrm{V}_{\mathrm{F}}{ }^{*}\right)$. Figure 5C shows that sampling rate also effects $\mathrm{V}_{\mathrm{F}}{ }^{*}$, with increased sampling rates resulting in increased measured $\mathrm{V}_{\mathrm{F}}{ }^{*}$. Figure 5D shows that acquisition pixel width also alters measured $\mathrm{V}_{\mathrm{F}}{ }^{*}$, with lower magnification (larger pixel with of $156 \mu \mathrm{m}$ ) increasing $\mathrm{V}_{\mathrm{F}}{ }^{*}$. Pixel binning however, did not alter $\mathrm{V}_{\mathrm{F}}{ }^{*}$.

We hypothesized that at smaller pixel sizes (highermagnification), the intrinsic AP upstroke within a single myocyte would play a more prominent role in determining AP upstroke morphology. If gap junction uncoupling by carbenoxolone does indeed increase $\mathrm{dV} / \mathrm{dt}_{\max }$, we would therefore expect at higher magnification a carbenoxolone induced increase in $\mathrm{dF} / \mathrm{dt}_{\max }$. However, data presented in Figure $5 \mathrm{E}$ show this not to be the case, as carbenoxolone was associated with a concentration-dependent decrease in optical AP upstroke velocity when recording at high-magnification from the ventricular free wall (20 $\mu \mathrm{m}$ pixel width) (Figure 5E). This is opposite to observed effects of gap junction inhibition on AP upstroke in microelectrode recordings but is in keeping with data presented in Figure 3 for lower-magnification optical mapping recordings (156 $\mu \mathrm{m}$ pixel width).

\section{Influence of Image and Signal Processing}

Figures 6A,B demonstrates the effects of spatial and temporal filtering on optical AP upstroke velocity. Figure 6A shows that application spatial filtering reduces $\mathrm{dF} / \mathrm{dt}_{\max }$. However, at kernel sizes of $5 \times 5$ pixels and larger (for the gaussian spatial filter applied herein), spatial filtering kernel size does not impact on measured $\mathrm{dF} / \mathrm{dt}_{\max }$. Temporal filtering reduces $\mathrm{dF} / \mathrm{dt}_{\max }$ in a frame size dependent manner. Figure 6C shows the effects of cubic spline interpolation at increasing effective sampling rates (from $1 \mathrm{kHz}$ acquisition sampling rate). $\mathrm{dF} / \mathrm{dt}_{\max }$ increases with 

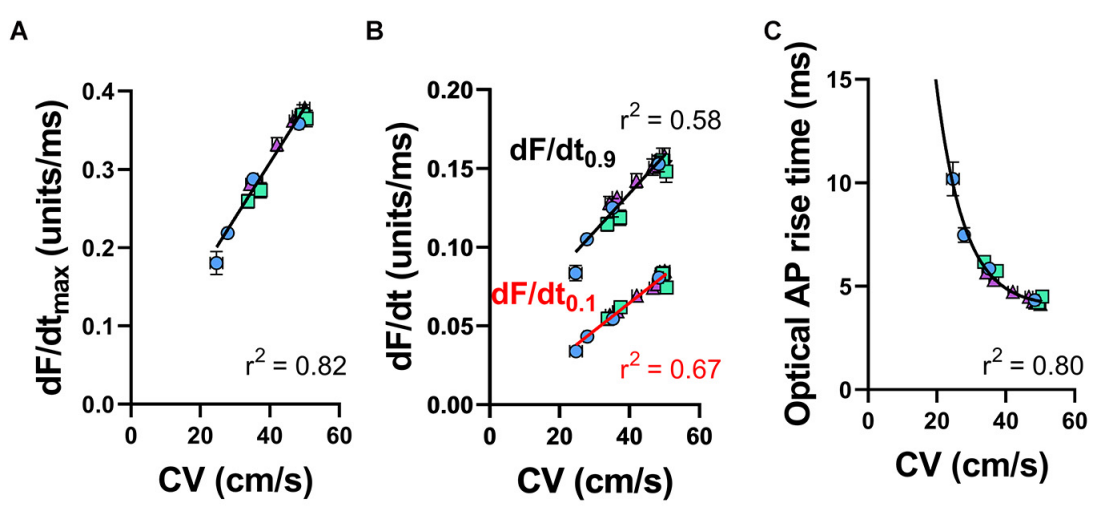

\section{- Flecainide $\quad \square$ Carbenoxo
$\Delta$ Ischaemia-reperfusion}

FIGURE 4 | Correlations of optical AP upstroke velocity, rise time and CV. (A) Correlation between CV and dF/dtmax of the optical AP upstroke. (B) Correlation between $\mathrm{CV}$ and $\mathrm{dF} / \mathrm{dt}_{0.9}$ (black) and dF/dt 0.1 (red) and of the optical AP upstroke. (C) Corresponding correlation between CV and optical AP rise times (measured between 0.1 and 0.9 fractional levels of AP upstroke). (A) Linear fit. (B) Exponential. Data are mean \pm SEM. Flecainide $n=7$ hearts. Carbenoxolone $n=7$ hearts. Ischemia-reperfusion $n=14$ hearts.

interpolation, however, interpolation to extreme high effective sampling rates (to $256 \mathrm{kHz}$ ) does not substantially alter measured $\mathrm{dF} / \mathrm{dt}_{\max }$ values from "moderate" interpolation up to $16 \mathrm{kHz}$.

\section{DISCUSSION}

It has been recognized for some time that the heterogeneity of activation of the myocardium is an important determinant of the rate of change and rise time of optically recorded APs (Morad and Salama, 1979; Girouard et al., 1996; Hyatt et al., 2003; Salama et al., 2005; Bishop et al., 2007) The present study is the first to compare the effects of interventions that slow cardiac conduction on the morphology of optical APs recorded from intact hearts. Our results show that optical AP upstroke velocity is sensitive to changes in local conduction due to low-flow ischemia, sodium channel blockade and gap junction inhibition. For all study interventions, the change in maximum AP upstroke velocity was found to be directly proportional to the corresponding change in tissue CV. This finding suggests that divergent mechanism-dependent effects, shown in previous studies using microelectrode recording techniques (Borchard and Boisten, 1982; Kojima et al., 1989; de Groot et al., 2003; Ferrero et al., 2007; Dhillon et al., 2013), do not alter effects of CV changes on optically recorded AP upstroke.

During ventricular pacing, we observed a clear anisotropic pattern in optical $\mathrm{dF} / \mathrm{dt}_{\max }$ maps, with more rapid AP upstroke velocities aligned along the direction of the most rapid rates of conduction (and vice versa). This observation differs from previous reports utilizing microelectrode recording techniques, where it is established that the rate of change of the AP upstroke is slowest along the fastest conduction path (Spach et al., 1987, 1988). The biophysical basis for this observation relates to differences in intracellular coupling along the longitudinal and transverse conduction paths. With greater coupling, as occurs in the longitudinal direction, more depolarizing current flows into neighboring cells (reduced source-sink ratio) (Spach et al., 1987, 1988). The net effect is faster CV but slower cellular AP upstroke velocity. The opposite is true in the transverse direction. This phenomenon was clearly explained in seminal studies by Spach et al. (1987, 1988). However, the results of our study demonstrate that such a relationship does not hold for optical mapping recordings, where it appears AP upstroke velocity is a linear function of local CV.

It is already known that whilst optical AP upstroke velocities in single cells are comparable to those recorded with microelectrodes, in intact tissue the upstroke of the optical AP is 2-5× slower (Girouard et al., 1996; Warren et al., 2010). Slowing of the optical AP upstroke is thought to arise from the summing of signals from a volume of tissue in which there are asynchronous activation times (see later discussion on photon scattering) (Girouard et al., 1996; Bishop et al., 2007). In this paradigm, slower $\mathrm{CV}$, leading to greater asynchrony in activation, would lead to a slower AP upstroke, in keeping with our experimental observations. Several findings from the present study support this interpretation. For instance, despite their differing mechanisms of action, $\mathrm{CV}$ and $\mathrm{dF} / \mathrm{dt}_{\max }$ values for ischemia, flecainide and carbenoxolone protocols all fall on a simple linear relationship. It has been suggested that gap junction inhibition slows $\mathrm{CV}$ in conjunction with a preserved or increased AP upstroke velocity, when recorded by microelectrode techniques. This has been reported in several studies and follows logically from the work of Dhillon et al., 2013; Spach et al., 1987, 1988; Thomas et al., 2003). There are however, several independent studies suggesting otherwise (Jalife et al., 1989; Ozaki et al., 1989; Rohr et al., 1998; Entz et al., 2016). In the present study, faster CV was associated with more rapid upstroke velocities in all interventions, strongly implying that local tissue $\mathrm{CV}$, and not tissue excitability (i.e., the intrinsic upstroke velocity of single myocytes), is the major determinant 
A

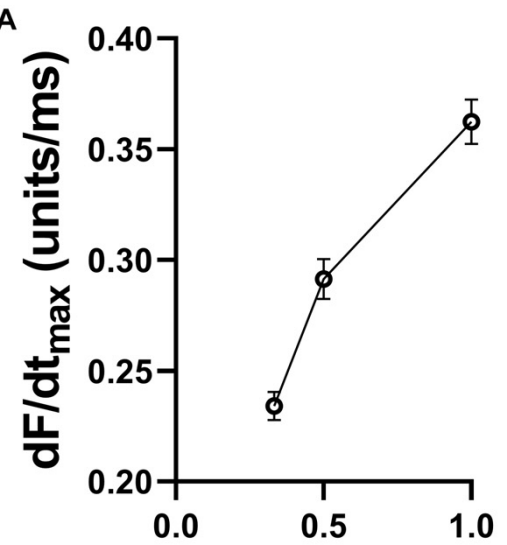

C

Sampling frequency $(\mathrm{kHz})$

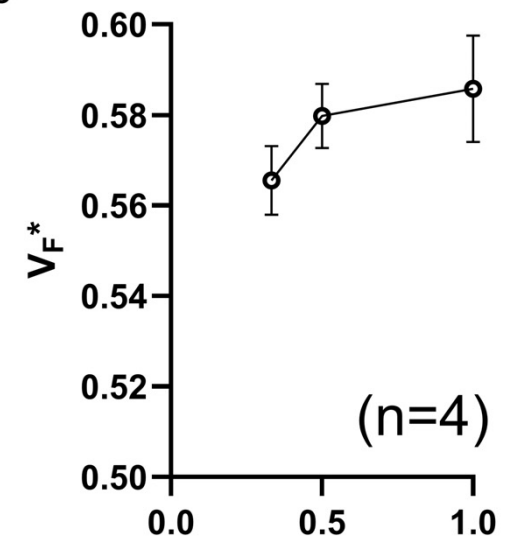

Sampling frequency $(\mathrm{kHz})$
B

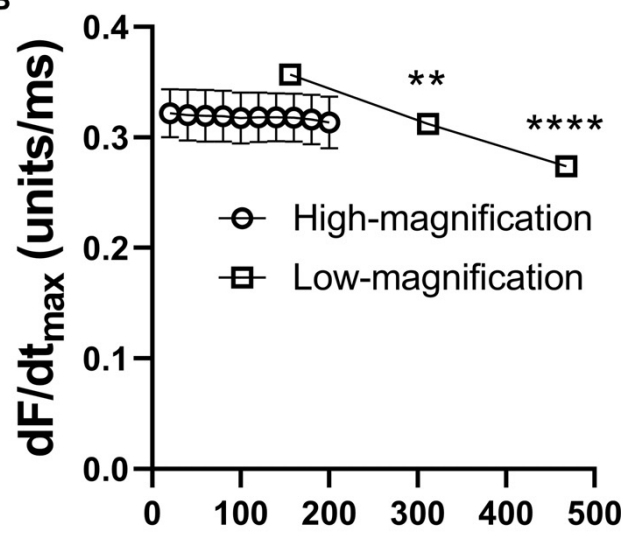

Pixel width $(\mu \mathrm{m})$

D

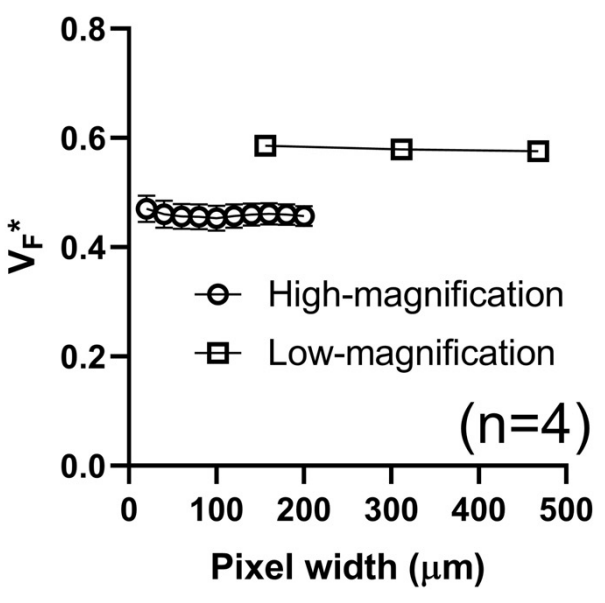

E

Pixel width $=20 \mu \mathrm{m}(\mathrm{n}=4)[1 \mathrm{kHz}]$

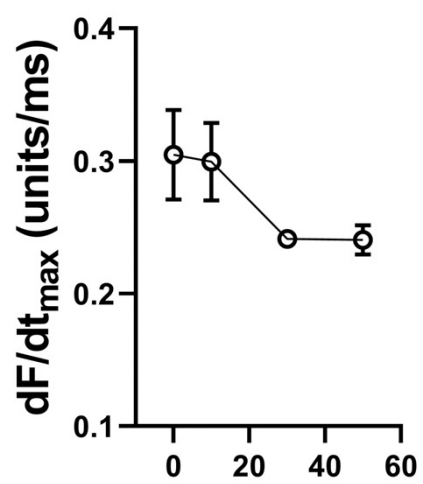

Carbenoxolone concentration ( $\mu \mathrm{mol} / \mathrm{L})$
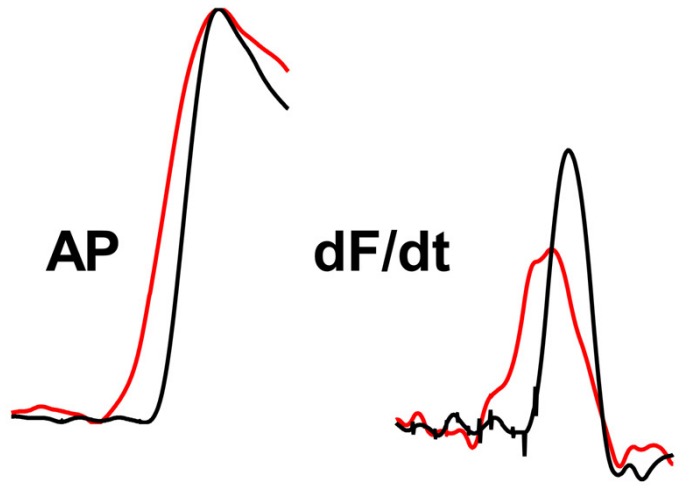

- Control

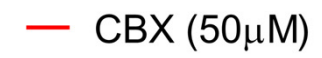

FIGURE 5 | Effects of sampling frequency, magnification and pixel binning. (A) Effects of altering acquisition sampling frequency on maximum optical AP upstroke velocity. (B) Effects of pixel-binning on AP upstroke velocity at low- and high-magnification (initial pixel widths of 156 and $20 \mu \mathrm{m}$ pixel width, respectively). (C) Effects of altering acquisition sampling frequency on $\bigvee_{F}^{*}$. (D) Effects of pixel-binning on AP upstroke velocity at low- and high-magnification on $V_{F}^{*}$. (E) Responses to carbenoxolone at high-magnification (20 $\mathrm{m}$ m pixel width). Data from 4 hearts with representative AP and dF/dt traces for control conditions and following carbenoxolone treatment $(50 \mu \mathrm{M})$. Other than post processing binning $(\mathrm{b}, \mathrm{d})$, all data presented here was processed with spatial filtering and interpolation (to $16 \mathrm{kHz})$ as set out in the methods. One-way ANOVA with Bonferroni post hoc tests. ${ }^{* *} p<0.01$ and ${ }^{* * * *} p<0.0001$. 

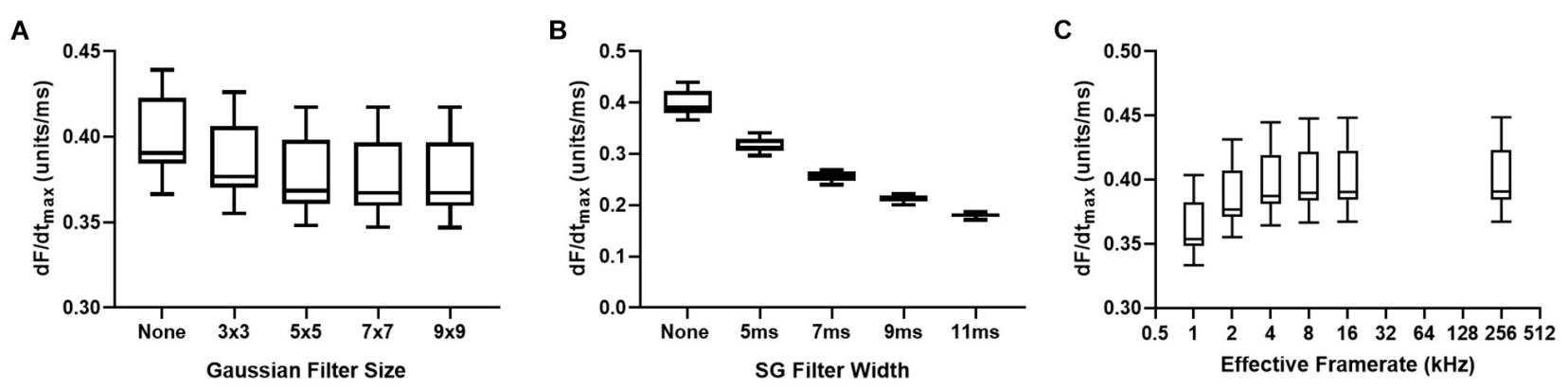

FIGURE 6 | Effects of data processing on maximum optical upstroke velocity (dF/dtmax). (A) Effect of gaussian spatial filter pixel size. (B) Effect of changing length/frame size of third order temporal Savitzky-Golay (SG) filter. (C) Effect of cubic spline interpolation to higher effective signal framerates. $n=14$ hearts.

of the upstroke velocity of optically recorded APs. Similar finding was found by Entz et al., 2016) in optically mapped intact guinea pig myocardium, with slowed $\mathrm{CV}$ induced by carbenoxolone resulting in prolonged optical rise times. Furthermore, this conclusion is indirectly supported by the findings of Hyatt et al. (2005) who found no correlation between microelectrode and optical AP upstroke velocities measured in the "same" region of isolated porcine right ventricle preparations. Our experimental results also corroborate computational predictions of a nonlinear relationship between the rise time of the optical AP and tissue CV (Hyatt et al., 2003).

Notably, we found that the above experimental observations remained true even when recording data at highermagnifications, with pixel widths as small as $20 \mu \mathrm{m}$, wherein carbenoxolone caused a comparable slowing of optical AP upstroke velocity as that observed in lower-magnification recordings. This is an interesting observation, as we originally hypothesized that the local AP upstroke (i.e., that of the individual myocytes) would become more dominant with smaller pixel widths, but we found this not to be the case. A likely explanation for this finding is the contribution of signals from within the ventricular wall, as well as the distortion of optical signals caused by fluorescent photon scattering within the tissue (Bishop et al., 2009). Illumination light, which excites membrane bound potentiometric dyes, penetrates in to cardiac tissue, and emitted fluorescent photons arising several hundred microns into the tissue contribute to the optical AP signal. Thus, the reduction in optical AP upstroke velocity with carbenoxolone, even when recording at high-magnification, may simply reflect the spatial differences in activation through the ventricular wall. Moreover, fluorescent photons undergo scattering events as they traverse to the tissue boundary, and due to these scattering events the photons detected on a single camera pixel originate from a widely distributed 3D tissue volume (Bishop et al., 2009). Computational models suggest that only a small proportion of the optical AP signal originates in tissues that are located geometrically beneath the recording pixel. Scattered photons contain information on the transmembrane potential at their site of origin, and so the recorded signal is the weighted average of the transmembrane potential levels within the scattered volume of tissue (Bishop et al., 2009). Our experimental data suggest that photon scattering effects dominate any local changes in AP upstroke in optical signals recorded from the mouse ventricle, even at high-magnification, and that this underpins the strong linear correlation between AP upstroke velocity and tissue CV. However, it is important again here to note the conflicting reports on the effects of gap junction uncoupling on the cellular AP upstroke, which may also explain our high-magnification findings (Jalife et al., 1989; Ozaki et al., 1989; Rohr et al., 1998; Entz et al., 2016). The mechanisms of camera tissue integration and photon scattering also explains why maximum $\mathrm{dF} / \mathrm{dt}$ was correlated with regions of fastest $\mathrm{CV}$, which diverges from the negative correlation observed for microelectrode recorded APs (Spach et al., 1987, 1988).

Ding et al. (2010) have previously reported on the use of maximum $\mathrm{dF} / \mathrm{dt}$ of the optical AP upstroke for quantification of differences in depolarization between normal and infarct borderzone in the heart of rats post myocardial infarction. Here the authors analyzed AP upstroke without controlling for differences in optical AP amplitude, which has its limitations. Optical AP amplitude depends not only on the absolute AP amplitude in cells under the recording site, but also heterogeneities in dye loading, regional illumination, and active tissue volume. Thus, it is unclear whether the reported differences in the study of Ding et al. (2010) reflect differences in local "excitability" or simply differences in dye loading/viable tissue within the border zone. On the contrary, amplitude-normalized signals may underestimate the magnitude of conduction slowing, in scenarios where a real change in AP amplitude plays a causal role in the slowing of CV (e.g., acute myocardial ischemia). Several studies have also reported on the related measure of AP rise time (Choi and Salama, 2000; Myles et al., 2010) though not in the context of quantifying electrical conduction in the heart, but rather as a comparison of AP kinetics with traditional microelectrode recordings. Computational modeling studies have predicted that optical AP rise time is a nonlinear function of $\mathrm{CV}$, which was confirmed in the present experiments (Hyatt et al., 2003). In studies of anisotropic conduction, Fast and Kléber reported no difference in $\mathrm{dV} / \mathrm{dt}$ (more accurately rescaled $\mathrm{dF} / \mathrm{dt}$ ) in optical AP recordings associated with longitudinal (slower) and transverse (faster) conduction in cultured neonatal myocyte monolayers (Fast and Kleber, 1994). This differs from the present studies observation of a correlation between anisotropic conduction 
patterns and $\mathrm{dF} / \mathrm{dt}$. The divergence in our results is not easily explained but may simply reflect the experimental model used (monolayers vs. intact hearts).

There has been substantive work on the contribution of subsurface signals to the morphology of the optical AP recorded in intact tissues. It is known that the fractional amplitude at which the optical AP upstroke has its maximal derivative $\left(\mathrm{V}_{\mathrm{F}}{ }^{*}\right)$ is a function of the subsurface orientation of electrical wavefronts in the heart (Hyatt et al., 2003, 2005, 2008; Zemlin et al., 2008). During epicardial pacing, this largely reflects the rotation of fibers in the ventricle, which is a major determinant of anisotropic conduction (Valderrabano, 2007). The results of the present study confirm these observations in the mouse heart, reproducing the typical $\mathrm{V}_{\mathrm{F}}{ }^{*}$ patterns expected for epicardial point stimulation (Hyatt et al., 2005; Zemlin et al., 2008).

\section{Study Limitations}

We show that AP upstroke velocity is dependent on the temporal sampling rate and spatial resolution, and for the same reason will be affected by signal processing techniques like spatial and temporal filtering and ensemble averaging. For example, we found that temporal filtering with a third order Savitizy-Goaly filter reduced baseline $\mathrm{dF} / \mathrm{dt}_{\max }$ values in a frame size dependent manner, and hence was not utilized. Thus, absolute AP upstroke is dependent on the experimental settings and data processing steps used. These considerations extend to use of interpolation to increase the effective sampling rate. Interpolation was applied in this study as the short timescale of the AP upstroke means $\mathrm{dF} / \mathrm{dt}_{\max }$ and $\mathrm{V}_{\mathrm{F}}{ }^{*}$ is likely to occur between sampling points at $1 \mathrm{kHz}$ sampling frequency ( $1 \mathrm{~ms}$ frame rate). $16 \mathrm{kHz}$ spline interpolation was applied as it was observed that lower effective frame rate reduced measured $\mathrm{dF} / \mathrm{dt}_{\max }$ values, while higher order interpolation (up to $256 \mathrm{kHz}$ ) did not change $\mathrm{dF} / \mathrm{dt}_{\max }$. However, the Nyquist theorem suggests that $1 \mathrm{kHz}$ sampling rate is only sufficient to accurately resolve signal frequency content less that $500 \mathrm{~Hz}$, meaning interpolation to $16 \mathrm{kHz}$ may be inaccurate. Furthermore, only one interpolation method (cubic spline) was tested. Hence, further investigation is required, potentially utilizing "ground truth" computer models of optical APs in intact tissue, to optimize data processing for measurement of maximum and fractional $\mathrm{dF} / \mathrm{dt}$.

The present studies were performed in isolated mouse hearts, and additional studies are required to establish the properties

\section{REFERENCES}

Bayly, P. V., KenKnight, B. H., Rogers, J. M., Hillsley, R. E., Ideker, R. E., and Smith, W. M. (1998). Estimation of conduction velocity vector fields from epicardial mapping data. IEEE Trans. Biomed. Eng. 45, 563-571. doi: 10.1109/10.668746

Bishop, M. J., Bub, G., Garny, A., Gavaghan, D. J., and Rodriguez, B. (2009). An investigation into the role of the optical detection set-up in the recording of cardiac optical mapping signals: a monte carlo simulation study. Physica D 238, 1008-1018. doi: 10.1016/j.physd.2008.06.014

Bishop, M. J., Gavaghan, D. J., Trayanova, N. A., and Rodriguez, B. (2007). Photon scattering effects in optical mapping of propagation and arrhythmogenesis in the heart. J. Electrocardiol. 40, S75-S80.

Borchard, U., and Boisten, M. (1982). Effect of flecainide on action potentials and alternating current-induced arrhythmias in mammalian myocardium. of the optical AP upstroke in other species. It seems highly probable that our findings in the mouse ventricle will be more broadly applicable, though the absolute relationship between optical AP upstroke $\mathrm{dF} / \mathrm{dt}_{\max }$ and tissue CV will be species- and setup- dependent.

\section{CONCLUSION}

In intact mouse hearts, slowing of optical AP upstroke velocity is directly proportional to the change in $\mathrm{CV}$ associated with low-flow ischemia, sodium channel block, and gap junction inhibition.

\section{DATA AVAILABILITY STATEMENT}

The datasets generated for this study are available on request to the corresponding author.

\section{ETHICS STATEMENT}

The animal study was reviewed and approved by the University of Birmingham Animal Welfare and Ethical Review Board.

\section{AUTHOR CONTRIBUTIONS}

JW conducted the majority of studies, developed the analysis software, and conducted the analysis. CO'S integrated the software into ElectroMap and conducted the analysis. KR and DP supervised CO'S. All authors wrote and reviewed the manuscript.

\section{FUNDING}

JW (FS/16/35/31952) was supported by the British Heart Foundation. DP and CO'S were supported by the (Sci-Phy4-Health Centre for Doctoral Training L016346) EPSRC and $(109604 / \mathrm{Z} / 15 / \mathrm{Z})$ Wellcome Trust and British Heart Foundation (PG/17/55/33087, RG/17/15/33106, FS/19/16/34169, and FS/19/12/34204). The Institute of Cardiovascular Sciences was supported by a BHF Accelerator Award, AA/18/2/34218.

J. Cardiovasc. Pharmacol. 4, 205-212. doi: 10.1097/00005344-19820300000007

Choi, B. R., and Salama, G. (2000). Simultaneous maps of optical action potentials and calcium transients in guinea-pig hearts: mechanisms underlying concordant alternans. J. Physiol. 529(Pt 1), 171-188. doi: 10.1111/j.1469-7793. 2000.00171.x

de Groot, J. R., Veenstra, T., Verkerk, A. O., Wilders, R., Smits, J. P., WilmsSchopman, F. J., et al. (2003). Conduction slowing by the gap junctional uncoupler carbenoxolone. Cardiovasc. Res. 60, 288-297. doi: 10.1016/j. cardiores.2003.07.004

Dhillon, P. S., Gray, R., Kojodjojo, P., Jabr, R., Chowdhury, R., Fry, C. H., et al. (2013). Relationship between gap-junctional conductance and conduction velocity in mammalian myocardium. Circ. Arrhythm. Electrophysiol. 6, 12081214. doi: 10.1161/CIRCEP.113.000848 
Ding, C., Gepstein, L., Nguyen, D. T., Wilson, E., Hulley, G., Beaser, A., et al. (2010). High-resolution optical mapping of ventricular tachycardia in rats with chronic myocardial infarction. Pacing Clin. Electrophysiol. 33, 687-695. doi: 10.1111/j.1540-8159.2010.02704.x

Entz, M. II, George, S. A., Zeitz, M. J., Raisch, T., Smyth, J. W., and Poelzing, S. (2016). Heart rate and extracellular sodium and potassium modulation of gap junction mediated conduction in guinea pigs. Front. Physiol. 7:16. doi: 10.3389/fphys.2016.00016

Fast, V. G., and Kleber, A. G. (1994). Anisotropic conduction in monolayers of neonatal rat heart cells cultured on collagen substrate. Circ. Res. 75, 591-595. doi: 10.1161/01.res.75.3.591

Ferrero, A., Chorro, F. J., Canoves, J., Mainar, L., Blasco, E., and Such, L. (2007). Effect of flecainide on longitudinal and transverse conduction velocities in ventricular myocardium. An experimental study. Rev. Esp. Cardiol. 60, $315-$ 318. doi: 10.1016/s1885-5857(07)60157-1

Girouard, S. D., Laurita, K. R., and Rosenbaum, D. S. (1996). Unique properties of cardiac action potentials recorded with voltage-sensitive dyes. J. Cardiovasc. Electrophysiol. 7, 1024-1038. doi: 10.1111/j.1540-8167.1996.tb00478.x

Hyatt, C. J., Mironov, S. F., Vetter, F. J., Zemlin, C. W., and Pertsov, A. M. (2005). Optical action potential upstroke morphology reveals near-surface transmural propagation direction. Circ. Res. 97, 277-284. doi: 10.1161/01.res.0000176022. 74579.47

Hyatt, C. J., Mironov, S. F., Wellner, M., Berenfeld, O., Popp, A. K., Weitz, D. A., et al. (2003). Synthesis of voltage-sensitive fluorescence signals from threedimensional myocardial activation patterns. Biophys. J. 85, 2673-2683. doi: 10.1016/s0006-3495(03)74690-6

Hyatt, C. J., Zemlin, C. W., Smith, R. M., Matiukas, A., Pertsov, A. M., and Bernus, O. (2008). Reconstructing subsurface electrical wave orientation from cardiac epi-fluorescence recordings: monte carlo versus diffusion approximation. Opt. Express 16, 13758-13772.

Jalife, J., Sicouri, S., Delmar, M., and Michaels, D. C. (1989). Electrical uncoupling and impulse propagation in isolated sheep Purkinje fibers. Am. J. Physiol. 257, H179-H189.

Klabunde, R. E. (2017). Cardiac electrophysiology: normal and ischemic ionic currents and the ECG. Adv. Physiol. Educ. 41, 29-37. doi: 10.1152/advan.00105. 2016

Kojima, M., Hamamoto, T., and Ban, T. (1989). Sodium channel-blocking properties of flecainide, a class IC antiarrhythmic drug, in guinea-pig papillary muscles. An open channel blocker or an inactivated channel blocker. Naunyn Schmiedebergs Arch. Pharmacol. 339, 441-447. doi: 10.1007/bf00736059

Morad, M., and Salama, G. (1979). Optical probes of membrane potential in heart muscle. J. Physiol. 292, 267-295. doi: 10.1113/jphysiol.1979.sp012850

Myles, R. C., Bernus, O., Burton, F. L., Cobbe, S. M., and Smith, G. L. (2010). Effect of activation sequence on transmural patterns of repolarization and action potential duration in rabbit ventricular myocardium. Am. J. Physiol. Heart Circ. Physiol. 299, H1812-H1822. doi: 10.1152/ajpheart.00518.2010

O’Shea, C., Holmes, A. P., Yu, T. Y., Winter, J., Wells, S. P., Correia, J., et al. (2019). ElectroMap: high-throughput open-source software for analysis and mapping of cardiac electrophysiology. Sci. Rep. 9:1389. doi: 10.1038/s41598-018-38 263-2

Ozaki, S., Nakaya, H., Gotoh, Y., Azuma, M., Kemmotsu, O., and Kanno, M. (1989). Effects of halothane and enflurane on conduction velocity and maximum rate of rise of action potential upstroke in guinea pig papillary muscles. Anesth. Analg. 68, 219-225.

Poelzing, S., and Rosenbaum, D. S. (2004). Altered connexin43 expression produces arrhythmia substrate in heart failure. Am. J. Physiol. Heart Circ. Physiol. 287, H1762-H1770.
Rohr, S., Kucera, J. P., and Kleber, A. G. (1998). Slow conduction in cardiac tissue, I: effects of a reduction of excitability versus a reduction of electrical coupling on microconduction. Circ. Res. 83, 781-794. doi: 10.1161/01.res.83.8.781

Salama, G., Choi, B. R., Azour, G., Lavasani, M., Tumbev, V., Salzberg, B. M., et al. (2005). Properties of new, long-wavelength, voltage-sensitive dyes in the heart. J. Membr. Biol. 208, 125-140. doi: 10.1007/s00232-005-0826-8

Salama, G., Kanai, A., and Efimov, I. R. (1994). Subthreshold stimulation of Purkinje fibers interrupts ventricular tachycardia in intact hearts. Experimental study with voltage-sensitive dyes and imaging techniques. Circ Res 74, 604-619. doi: 10.1161/01.res.74.4.604

Spach, M. S., Dolber, P. C., and Heidlage, J. F. (1988). Influence of the passive anisotropic properties on directional differences in propagation following modification of the sodium conductance in human atrial muscle. A model of reentry based on anisotropic discontinuous propagation. Circ. Res. 62, 811-832. doi: 10.1161/01.res.62.4.811

Spach, M. S., Dolber, P. C., Heidlage, J. F., Kootsey, J. M., and Johnson, E. A. (1987). Propagating depolarization in anisotropic human and canine cardiac muscle: apparent directional differences in membrane capacitance. A simplified model for selective directional effects of modifying the sodium conductance on Vmax, tau foot, and the propagation safety factor. Circ. Res. 60, 206-219. doi: 10.1161/01.res.60.2.206

Stanley, W. C. (2001). Changes in cardiac metabolism: a critical step from stable angina to ischaemic cardiomyopathy. Eur. Heart J. Suppl. 3, O2-O7.

Thomas, S. P., Kucera, J. P., Bircher-Lehmann, L., Rudy, Y., Saffitz, J. E., and Kleber, A. G. (2003). Impulse propagation in synthetic strands of neonatal cardiac myocytes with genetically reduced levels of connexin43. Circ. Res. 92, 1209-1216. doi: 10.1161/01.res.0000074916.412 21.ea

Valderrabano, M. (2007). Influence of anisotropic conduction properties in the propagation of the cardiac action potential. Prog. Biophys. Mol. Biol. 94, 144168. doi: 10.1016/j.pbiomolbio.2007.03.014

Walton, R. D., Smith, R. M., Mitrea, B. G., White, E., Bernus, O., and Pertsov, A. M. (2012). Extracting surface activation time from the optically recorded action potential in three-dimensional myocardium. Biophys. J. 102, 30-38. doi: 10.1016/j.bpj.2011.10.036

Warren, M., Spitzer, K. W., Steadman, B. W., Rees, T. D., Venable, P., Taylor, T., et al. (2010). High-precision recording of the action potential in isolated cardiomyocytes using the near-infrared fluorescent dye di-4-ANBDQBS. Am. J. Physiol. Heart Circ. Physiol. 299, H1271-H1281. doi: 10.1152/ajpheart.00248. 2010

Windisch, H., Müller, W., and Tritthart, H. A. (1985). Fluorescence monitoring of rapid changes in membrane potential in heart muscle. Biophys. J. 48, 877-884. doi: 10.1016/s0006-3495(85)83849-2

Zemlin, C. W., Bernus, O., Matiukas, A., Hyatt, C. J., and Pertsov, A. M. (2008). Extracting intramural wavefront orientation from optical upstroke shapes in whole hearts. Biophys. J. 95, 942-950. doi: 10.1529/biophysj.107.117887

Conflict of Interest: The authors declare that the research was conducted in the absence of any commercial or financial relationships that could be construed as a potential conflict of interest.

Copyright (c) 2019 O'Shea, Pavlovic, Rajpoot and Winter. This is an open-access article distributed under the terms of the Creative Commons Attribution License (CC BY). The use, distribution or reproduction in other forums is permitted, provided the original author(s) and the copyright owner(s) are credited and that the original publication in this journal is cited, in accordance with accepted academic practice. No use, distribution or reproduction is permitted which does not comply with these terms. 